\title{
Perfil sociodemográfico e metabólico de adolescentes nascidos a termo e pré-termo
}

\author{
Sociodemographic and metabolic profile of term and preterm adolescents \\ Perfil sociodemográfico y metabólico de adolescentes a término y prematuros
}

Recebido: 30/01/2022 | Revisado: 03/02/2022 | Aceito: 11/02/2022 | Publicado: 17/02/2022

Fabíula dos Santos Toso

ORCID: https://orcid.org/0000-0003-1974-6921

Universidade Estadual do Oeste do Paraná. Brasil

E-mail: fabitoso@hotmail.com

Cláudia Silveira Viera

ORCID: https://orcid.org/0000-0002-0900-4660

Universidade Estadual do Oeste do Paraná. Brasil

E-mail: clausviera@gmail.com

Ana Tereza Bittencourt Guimarães

ORCID: https://orcid.org/0000-0002-3633-6484

Universidade Estadual do Oeste do Paraná. Brasil

E-mail: anatbguimaraes@gmail.com

\begin{abstract}
Resumo
Este estudo tem como objetivo identificar e descrever o perfil sociodemográfico, antropométrico, pressórico, glicêmico e lipídico de adolescentes nascidos a termo e pré-termo. Estudo de abordagem quantitativa, de desenho transversal, associado a análise de dados primários e secundários. A amostragem foi por conveniência, composta por 150 adolescentes entre 10 e 19 anos de idade, nascidos a termo, e 50 adolescentes nascidos prematuros. Realizaram-se entrevistas e avaliação física dos adolescentes (aferição da pressão arterial, mensuração antropométrica, circunferência abdominal e coleta de sangue por punção capilar para exames de glicose, colesterol total e triglicerídeos). Para análise estatística utilizou-se o teste de Qui-quadrado para independência $(\alpha=0,05)$. A maioria dos adolescentes tinha idade entre 10 a 13 anos e autorreferidos como brancos. Os pais apresentavam 7 a 12 anos de estudo e possuíam vínculo empregatício. O consumo alimentar mais saudável foi detectado entre os adolescentes prematuros, com aleitamento materno acima de seis meses para maioria em ambos os grupos. Foi identificado realização de atividades físicas com frequência inadequada. A maioria é composta por indivíduos eutróficos, contudo, $34 \%$ dos nascidos a termo e $30 \%$ dos prematuros estavam com sobrepeso ou obesos. Não foram verificadas diferenças entre os perfis antropométrico, pressórico e glicêmico entre os grupos.
\end{abstract}

Palavras-chave: Saúde do adolescente; Prematuridade; Promoção da saúde escolar.

\begin{abstract}
This study aims to identify and describe the sociodemographic, anthropometric, pressure, glycemic and lipid profile of term and preterm adolescents. Study of quantitative approach, cross-sectional design, associated with analysis of primary and secondary data. The sampling consisted of 150 adolescents between 10 and 19 years of age, born at term, and 50 adolescents born prematurely. Interviews and physical evaluation of adolescents were conducted (blood pressure measurement, anthropometric measurement, abdominal circumference and blood collection by capillary puncture for glucose, total cholesterol and triglyceride scans). For statistical analysis, the Chi-square test for independence $(\alpha=0.05)$ was used. Most adolescents were between 10 and 13 years old and self-reported as white. The parents had 7 to 12 years of study and had employment. The healthiest food intake was detected among premature adolescents, with breastfeeding above six months for the majority in both groups. Physical activities were identified with inadequate frequency. The majority are eutrophic individuals, however, $34 \%$ of full-term and $30 \%$ of preterm infants were overweight or obese. There were no differences between anthropometric, blood pressure and glycemic profiles between the groups.
\end{abstract}

Keywords: Adolescent health; Prematurity; Promotion of school health.

\section{Resumen}

Este estudio tiene como objetivo identificar y describir el perfil sociodemográfico, antropométrico, de presión, glucémico y lipídico de adolescentes a término y prematuros. Estudio de enfoque cuantitativo, diseño transversal, asociado con el análisis de datos primarios y secundarios. La muestra consistió en 150 adolescentes entre 10 y 19 años de edad, nacidos a término, y 50 adolescentes nacidos prematuramente. Se realizaron entrevistas y evaluación física de adolescentes (medición de la presión arterial, medición antropométrica, circunferencia abdominal y extracción de sangre por punción capilar para gammagrafías de glucosa, colesterol total y triglicéridos). Para el análisis estadístico se utilizó la prueba de Chi-cuadrado para la independencia $(\alpha=0,05)$. La mayoría de los adolescentes tenían entre 10 y 13 años de edad y se autodenominaban blancos. Los padres tenían de 7 a 12 años de estudio y tenían empleo. La 
ingesta de alimentos más saludable se detectó entre los adolescentes prematuros, con lactancia materna por encima de los seis meses para la mayoría en ambos grupos. Las actividades físicas se identificaron con frecuencia inadecuada. La mayoría son individuos eutróficos, sin embargo, el 34\% de los bebés prematuros a término y el $30 \%$ tenían sobrepeso u obesidad. No hubo diferencias entre los perfiles antropométricos, de presión arterial y glucémicos entre los grupos.

Palabras clave: Salud del adolescente; Prematuridad; Promoción de la salud escolar.

\section{Introdução}

Os partos prematuros mundialmente vêm evidenciando gradativo aumento, visto que, em 2000, correspondiam a 9,8\% dos nascimentos, no ano de 2014, a 10,6\% (Chawanpaiboon, et al., 2019) e em 2016 os nascidos pré-termo (PT) representaram $11,11 \%$ do total de nascidos vivos (Sinasc, 2019). No Brasil, entre os anos de 2013 a 2018, o índice de prematuridade médio foi de $11,1 \%$, apresentando semelhanças regionais (Brasil, 2021).

Com esse aumento, tem-se também que a prematuridade foi responsável por $17,1 \%$ dos óbitos infantis e $29,3 \%$ dos óbitos no primeiro mês de vida, em 2017, no Brasil (Unicef, 2019). Esses dados apontam a importância da assistência adequada à gestante e ao recém-nascido $(\mathrm{RN})$, durante o período pré-natal, parto e atendimento imediato à criança, para evitar os óbitos neonatais (Sinasc, 2019). Além disso, tais condutas reduzem o risco de sequelas, visto que a prematuridade pode levar a várias complicações, no período neonatal, como também em longo prazo, sendo associada ao maior risco de desenvolvimento de doenças crônicas, a exemplo de problemas pulmonares, cardiovasculares e metabólicos (Luu, et al., 2016).

Essas complicações podem também ser observadas na adolescência, que caracteriza-se como fase evolutiva de intensas transformações físicas e sociais antecedentes a fase adulta, compreendendo a faixa etária aproximadamente entre os 10 e 19 anos de idade (WHO, 2017). A adolescência juntamente ao período fetal e neonatal se configuram como períodos de maior risco à exposição externas e internas, o que podem levar a alterações metabólicas e a doenças crônicas na vida adulta, fase denominada de janela crítica (Burggren \& Mueller, 2015). A prematuridade se constitui, portanto, em um fator de risco a que o feto foi submetido e, consequentemente, poderá ter exposto esse indivíduo a distintas alterações, como a alteração metabólica no período da adolescência.

Associadas aos aspectos supracitados, têm-se as mudanças no estilo de vida entre os adolescentes, com redução de realizações de atividades físicas, fator vinculado ao aumento do consumo de alimentos com elevado teor de gorduras, sódio e açúcares (Carneiro, et al., 2017). Ademais, a diminuição no consumo de alimentos saudáveis acarreta aumento na prevalência de excesso de peso e obesidade nessa fase. Associações com sexo, nível socioeconômico e hereditariedade também são determinantes para a origem da obesidade em adolescentes (SBD, 2019). Esta está associada a complicações como Diabete Mellitus tipo 2, Hipertensão arterial sistêmica (HAS) e dislipidemias, que corroboram ao aumento do risco de eventos cardiovasculares (SBD, 2019).

Embasando-se nessas evidências, acredita-se que adolescentes nascidos prematuramente encontram-se mais vulneráveis a desenvolverem problemas cardiovasculares, obesidade e diabetes do que aqueles nascidos a termo (AT). Desse modo, é pertinente identificar e descrever o perfil sociodemográfico, antropométrico, pressórico, glicêmico e lipídico de adolescentes nascidos AT e PT, em busca de caracterizar ambos os grupos e verificar se esses apresentam diferenças em relação aos perfis analisados, possibilitando assim o manejo adequado da saúde do adolescente para prevenir o risco de condições crônicas na vida adulta.

\section{Metodologia}

Estudo de abordagem quantitativa, de desenho transversal, associado a análise de dados primários e secundários (Pereira, et al., 2018, Cheng \& Phillips, 2014 \& Vezzoni, 2015), desenvolvido em município da região oeste do Paraná. A amostragem foi por conveniência, composta por 150 adolescentes entre 10 e 19 anos de idade, nascidos AT, e 50 adolescentes 
nascidos PT. Como critérios de exclusão, tivemos a idade e ausência de autorização dos responsáveis, mediante o Termo de Compromisso Livre e Esclarecido.

Os adolescentes do grupo AT foram captados em escola do município e os PT foram obtidos a partir de banco de dados secundário, de pesquisa realizada por Lopes (2018) na mesma região do município.

As técnicas de coleta de dados seguiram a metodologia proposta no estudo primário, as quais envolveram, em primeiro momento, a realização de entrevistas, com auxílio de pesquisadores previamente capacitados, por meio de formulário estruturado, elaborado por Lopes (2018), sendo analisadas as variáveis: idade, cor auto-declarada, idade dos pais, escolaridade e ocupação dos mesmos, renda familiar, número de dependentes e participação em programas de transferência de renda (Programa Bolsa Família - PBF ou outros), idade gestacional (IG) de nascimento e aleitamento materno. Estes últimos solicitados aos pais, em formulário entregue previamente. Os aspectos biopsicossociais incluíram hábitos de lazer e realização de atividades físicas. Os marcadores de consumo alimentar foram analisados conforme o Sistema de Vigilância Alimentar e Nutricional (SISVAN), incluindo hábitos de realizar refeições frente às telas ou não, número de refeições e consumo alimentar.

Após a entrevista, os adolescentes foram submetidos à avaliação física sumária, que compreendeu a aferição da pressão arterial (PA), verificada com o adolescente sentado e após 10 minutos de repouso, mensurada duas vezes com intervalo de cinco minutos e calculada a média da PA. A análise dessa variável deu-se pela identificação do percentil das pressões arteriais diastólicas e sistólicas médias, (PADM e PASM, respectivamente) (SBC, 2005), classificadas por meio de gráfico de percentil da altura por idade e sexo. É considerada como PA elevada quando se encontra acima do P90. Para adolescentes de 17 a 19 anos, a classificação seguiu os parâmetros de adultos (SBC, 2005), sendo alterada, quando PASM > 120 e PADM > 80.

Ainda, fez-se a mensuração antropométrica (peso e estatura) e da circunferência abdominal (CA), sendo que, para a avaliação do peso, o adolescente encontrava-se descalço, com pés afastados, pouca roupa, posição ereta, sendo a medida registrada em quilogramas $(\mathrm{Kg})$. A avaliação da estatura foi registrada em centímetros $(\mathrm{cm})$ e realizada com o adolescente descalço, com o calcanhar, glúteo e a cabeça encostados na régua, joelhos esticados e o olhar direcionado à linha do horizonte. A análise foi baseada no Escore-Z do Índice de Massa Corpórea (IMC), recomendados pela Organização Mundial da Saúde e utilizados pelo Ministério da Saúde do Brasil. O IMC foi classificado conforme o sexo, sendo considerado eutrófico $(-2<$ escore $Z<+1)$, magreza $(-3<$ escore $Z<-2$ ), magreza acentuada (escore $Z<-3$ ), sobrepeso $(+1<$ escore $Z<+2)$ e obeso (escore Z > +2) (Brasil, 2012).

Em seguida, foi realizada a medida da CA, com uso de fita métrica inelástica, graduada em milímetros, sendo a medida no ponto médio entre a borda inferior da última costela e a margem superior da crista ilíaca. Os dados foram avaliados com base na International Diabetes Federation (IDF, 2006), classificados como elevada, quando Percentil $\geq 90$.

Posteriormente, foi realizada a coleta de sangue por punção capilar para exames bioquímicos de glicose, colesterol total (CT) e triglicerídeos (TG). A análise dos resultados foi realizada com base na Sociedade Brasileira de Diabetes (SBD, 2019), sendo a glicemia classificada como elevada se $\geq 100$ (jejum) ou $\geq 140$ (pós ingestão alimentar).

Conforme as especificações do fabricante - Roche Diagnostics $\mathrm{GmbH}$, o resultado do exame de $\mathrm{CT}$ foi apresentado no intervalo de medição de 150-300mg/dL, com coeficiente de variação de 0,8 a 3,7\% (Roche, 2014a). Já o exame de TG foi apresentado no intervalo de medição de $70-600 \mathrm{mg} / \mathrm{dL}$, com coeficiente de variação de 3,1 a 3,4\%, indicado pelo mesmo fabricante (Roche, 2014b). Os resultados foram avaliados com base nos valores de referência da Sociedade Brasileira de Análises Clínicas (SBAC, 2016), classificados como elevado quando TG $\geq 90 \mathrm{mg} / \mathrm{dl}$ (jejum) ou $\geq 100 \mathrm{mg} / \mathrm{dl}$ (não jejum) e CT $\geq$ $170 \mathrm{mg} / \mathrm{dl}$.

A tabulação de dados dos adolescentes nascidos AT foi realizada em banco de dados no programa Microsoft Excel®, por digitação, com conferência dupla, para posterior comparação ao banco de dados secundário, de adolescentes nascidos 
prematuros. Para identificar o perfil da amostra, empregou-se a estatística descritiva, em que as variáveis foram analisadas em dois grupos, adolescentes nascidos AT e PT, por meio de frequências absolutas e relativas percentuais. Em seguida, a análise de significância foi realizada por meio do teste de Qui-quadrado para Independência. As análises foram realizadas com $\alpha=5 \%$ de significância estatística, com os programas licenciados XLSTat versão 2017 (Addinsoft, 2017) e R (R Development Core Team, 2019).

Todos os preceitos éticos foram seguidos, em que os adolescentes participantes assinaram o Termo de Assentimento Livre e Esclarecido e os pais/responsáveis autorizaram a participação, por meio de um Termo de Consentimento Livre e Esclarecido. O estudo foi aprovado pelo Comitê de Ética e Pesquisa com Seres Humanos da Unioeste, sob o parecer $n^{\circ}$ 2.625.378.

\section{Resultados}

A tabela 1 apresenta a caracterização de adolescentes nascidos a termo e pré-termo. Os grupos AT e o PT, em estudo, caracterizam-se por adolescentes, em sua maioria, com idade entre 10 e 13 anos, que se autodeclararam de etnia branca. Com relação à caracterização ao sexo biológico, o grupo AT, em sua maioria, era de adolescentes do sexo feminino e no grupo PT, do sexo masculino ( $\mathrm{p}>0,05)$.

Quanto ao tipo de atividade de lazer, a maioria, foi classificada como lazer ativo, atividades que envolvem maior movimentação corporal, como esportes (AT 69\% e PT 62\%), apresentando discreta elevação no grupo AT, em comparação ao grupo PT. Assim como, dentre os que realizam atividades de lazer passivo (sedentárias), como uso de computadores, televisão, videogame, foi superior no grupo PT (36\%) em comparação ao grupo AT $(25 \%)(\mathrm{p}=0,1751)$.

Com relação à frequência de atividades físicas, a maioria se enquadrou na categoria inadequada (atividades físicas $<3$ vezes/semana), porém, a frequência do grupo PT foi significativamente superior em comparação ao grupo AT (p-valor = 0,0058), considerando-se, portanto, o grupo PT mais sedentário. Salienta-se que, neste estudo, foram analisados somente a frequência semanal de atividades físicas e o tipo de atividade realizada, não sendo analisado o tempo destinado para tal atividade.

Dentre os hábitos alimentares, classificou-se, primeiramente, conforme o hábito de uso de telas durante as refeições, sendo que a maioria foi classificada como intermediária, ou seja realizavam igual ou mais de 4 refeições/dia em frente às telas ou realizavam de 2 a 4 refeições/dia não em frente às telas, apresentando discreta elevação destas frequências do grupo PT. Na classificação inadequada, o grupo AT foi superior ao PT ( $p>0,05)$.

Quanto ao tipo de alimentação, em sua maioria os adolescentes foram classificados na categoria intermediária (consumo moderado de alimentos saudáveis e não saudáveis), com frequências semelhantes em ambos os grupos ( $p>0,05)$. A maioria dos adolescentes recebeu aleitamento materno, com uma frequência maior no grupo AT. Foi predominante entre os grupos o tempo de amamentação acima de seis meses de idade, com discreta elevação no grupo PT, em comparação ao grupo AT (p>0,05). 
Tabela 1 - Caracterização de adolescentes nascidos a termo (N=150) e pré-termo (N=50). Cascavel-PR, Brasil, 2019.

\begin{tabular}{|c|c|c|c|c|}
\hline Variáveis & Categorias & $\begin{array}{l}\text { A Termo- } \\
\text { FA }(\text { FR\% })\end{array}$ & $\begin{array}{c}\text { Pré-termo - } \\
\text { FA }(\text { FR\% })\end{array}$ & p-valor \\
\hline \multirow{3}{*}{ Idade } & Entre 10 a 13 anos & $81(54 \%)$ & $27(54 \%)$ & \multirow{3}{*}{0,3247} \\
\hline & $>13$ a 16 anos & $53(35 \%)$ & $17(34 \%)$ & \\
\hline & $>16$ anos & $16(11 \%)$ & $6(12 \%)$ & \\
\hline \multirow{2}{*}{ Sexo } & Masculino & $58(39 \%)$ & $26(52 \%)$ & \multirow{2}{*}{0,0981} \\
\hline & Feminino & $92(61 \%)$ & $24(48 \%)$ & \\
\hline \multirow{3}{*}{ Cor } & Branca & $84(56 \%)$ & $27(54 \%)$ & \multirow{3}{*}{0,5581} \\
\hline & Parda & $63(42 \%)$ & $23(46 \%)$ & \\
\hline & Negra & $3(2 \%)$ & $0(0 \%)$ & \\
\hline \multirow{3}{*}{ Lazer } & Ativo & $103(69 \%)$ & $31(62 \%)$ & \multirow{3}{*}{0,1751} \\
\hline & Passivo & $37(25 \%)$ & $18(36 \%)$ & \\
\hline & Não informado & $10(7 \%)$ & $1(2 \%)$ & \\
\hline \multirow{2}{*}{ Adequação de Atividades físicas } & Adequada & $59(39 \%)$ & $9(18 \%)$ & \multirow{2}{*}{0,0058} \\
\hline & Inadequada & $91(61 \%)$ & $41(82 \%)$ & \\
\hline \multirow{3}{*}{ Hábitos telas/refeições } & Ideal & $12(8 \%)$ & $3(6 \%)$ & \multirow{3}{*}{0,4818} \\
\hline & Intermediário & $107(71 \%)$ & $40(80 \%)$ & \\
\hline & Inadequado & $31(21 \%)$ & $7(14 \%)$ & \\
\hline \multirow{3}{*}{ Tipo de alimentação } & Ideal & $24(16 \%)$ & $10(20 \%)$ & \multirow{3}{*}{0,7562} \\
\hline & Intermediário & $94(63 \%)$ & $31(62 \%)$ & \\
\hline & Inadequado & $32(21 \%)$ & $9(18 \%)$ & \\
\hline \multirow{3}{*}{ Aleitamento materno } & Não & $11(7 \%)$ & $8(16 \%)$ & \multirow{3}{*}{0,108} \\
\hline & Sim & $135(90 \%)$ & $42(84 \%)$ & \\
\hline & Não informado & $4(3 \%)$ & $0(0 \%)$ & \\
\hline \multirow{4}{*}{ Tempo aleitamento materno } & Não se aplica & $20(13 \%)$ & $10(20 \%)$ & \multirow{4}{*}{0,462} \\
\hline & 0 a 4 meses & $39(26 \%)$ & $9(18 \%)$ & \\
\hline & 4 a 6 meses & $23(15 \%)$ & $6(12 \%)$ & \\
\hline & $>6$ meses & $68(45 \%)$ & $25(50 \%)$ & \\
\hline
\end{tabular}

FA: Frequência absoluta; FR: Frequência relativa. Fonte: Dados da pesquisa e Lopes (2018).

A Tabela 2 apresenta o perfil sociodemográfico de adolescentes nascidos a termo e pré-termo. Evidenciou-se que a maioria das mães e pais cursou de 7 a 12 anos de estudo. No entanto, no grupo PT, os pais que apresentaram acima de 12 anos de estudo foram em maior número, em comparação ao grupo AT, tanto para as mães $(\mathrm{p}=0,0004)$ como para os pais $(\mathrm{p}$ $<0,0001)$.

A maioria das mães tinha vínculo empregatício, com maior proporção no grupo PT ( $\mathrm{p}=0,2316)$. O grupo AT apresentou maior número de mães classificadas como do lar ( $\mathrm{p}>0,05)$. Os pais, em sua maioria, também tinham vínculo empregatício, com maior frequência no grupo PT, seguidos da classificação autônomos, com maior frequência verificada no grupo AT $(\mathrm{p}=0,0399)$.

O estado civil dos pais foi predominante como casados com renda familiar entre 2 a 3 salários mínimos. O grupo PT, porém, apresentou maior frequência para quatro salários mínimos ou mais, continha de 3 a 5 pessoas dependentes no domicílio e a maioria das famílias não era beneficiária do $\operatorname{PBF}(\mathrm{p}>0,05$; Tabela 2). A maioria também não participava de outros programas sociais, mas as pessoas que participavam estavam em maior frequência, no grupo PT $(\mathrm{p}=0,0167)$. 
Tabela 2 - Perfil sociodemográfico de adolescentes nascidos a termo (N=150) e pré-termo (N=50). Cascavel-PR, Brasil, 2019.

\begin{tabular}{|c|c|c|c|c|}
\hline Variáveis & Categorias & $\begin{array}{l}\text { A Termo- } \\
\text { FA }(\text { FR\%) }\end{array}$ & $\begin{array}{c}\text { Pré-termo - FA } \\
(\text { FR\%) }\end{array}$ & p-valor \\
\hline \multirow{5}{*}{ Escolaridade Mãe } & Não se aplica & $21(14 \%)$ & $0(0 \%)$ & \multirow{5}{*}{0,0004} \\
\hline & $<5$ anos & $11(7 \%)$ & $0(0 \%)$ & \\
\hline & 5 a 6 anos & $19(13 \%)$ & $6(12 \%)$ & \\
\hline & 7 a 12 anos & $87(58 \%)$ & $31(62 \%)$ & \\
\hline & $>12$ anos & $12(8 \%)$ & $13(26 \%)$ & \\
\hline \multirow{8}{*}{ Ocupação mãe } & Do lar & $41(27 \%)$ & $9(18 \%)$ & \multirow{8}{*}{0,2316} \\
\hline & Com vínculo empregatício & $71(47 \%)$ & $31(62 \%)$ & \\
\hline & Autônoma & $22(15 \%)$ & $7(14 \%)$ & \\
\hline & Desempregado & $6(4 \%)$ & $0(0 \%)$ & \\
\hline & Serviço público & $4(3 \%)$ & $3(6 \%)$ & \\
\hline & Outros & $0(0 \%)$ & $0(0 \%)$ & \\
\hline & Aposentado/pensionista & $2(1 \%)$ & $0(0 \%)$ & \\
\hline & Não sabe/NA** & $4(3 \%)$ & $0(0 \%)$ & \\
\hline \multirow{5}{*}{ Escolaridade pai } & Não se aplica & $54(36 \%)$ & $4(8 \%)$ & \multirow{5}{*}{$<0,0001$} \\
\hline & $<5$ anos & $8(5 \%)$ & $3(6 \%)$ & \\
\hline & 5 a 6 anos & $29(19 \%)$ & $7(14 \%)$ & \\
\hline & 7 a 12 anos & $57(38 \%)$ & $28(56 \%)$ & \\
\hline & $>12$ anos & $2(1 \%)$ & $8(16 \%)$ & \\
\hline \multirow{7}{*}{ Ocupação pai } & Com vínculo empregatício & $60(40 \%)$ & $31(62 \%)$ & \multirow{7}{*}{0,0399} \\
\hline & Autônoma & $56(37 \%)$ & $14(28 \%)$ & \\
\hline & Desempregado & $4(3 \%)$ & $0(0 \%)$ & \\
\hline & Serviço público & $4(3 \%)$ & $0(0 \%)$ & \\
\hline & Outros & $0(0 \%)$ & $1(2 \%)$ & \\
\hline & Aposentado/pensionista & $4(3 \%)$ & $0(0 \%)$ & \\
\hline & Não sabe/NA** & $22(15 \%)$ & $4(8 \%)$ & \\
\hline \multirow{5}{*}{ União pais } & Casados & $93(62 \%)$ & $31(62 \%)$ & \multirow{5}{*}{0,3685} \\
\hline & Separados & $46(31 \%)$ & $19(38 \%)$ & \\
\hline & Viúvos & $7(5 \%)$ & $0(0 \%)$ & \\
\hline & Solteiros & $1(1 \%)$ & $0(0 \%)$ & \\
\hline & Não sabe/NA** & $3(2 \%)$ & $0(0 \%)$ & \\
\hline \multirow{3}{*}{ Renda } & Até um SM & $16(11 \%)$ & $7(14 \%)$ & \multirow{3}{*}{0,1491} \\
\hline & 2 a 3 SM & $106(71 \%)$ & $28(56 \%)$ & \\
\hline & 4 ou mais SM & $28(19 \%)$ & $15(30 \%)$ & \\
\hline \multirow{3}{*}{ Número dependentes } & Até 2 pessoas & $8(5 \%)$ & $3(6 \%)$ & \multirow{3}{*}{0,6953} \\
\hline & 3 a 5 pessoas & $122(81 \%)$ & $38(76 \%)$ & \\
\hline & $>5$ pessoas & $20(13 \%)$ & $9(18 \%)$ & \\
\hline \multirow{2}{*}{ Programa Bolsa Família } & Não & $130(87 \%)$ & $46(92 \%)$ & \multirow{2}{*}{0,3149} \\
\hline & Sim & $20(13 \%)$ & $4(8 \%)$ & \\
\hline \multirow{2}{*}{ Outros Programas Sociais } & Não & $148(99 \%)$ & $46(92 \%)$ & \multirow{2}{*}{0,0167} \\
\hline & Sim & $2(1 \%)$ & $4(8 \%)$ & \\
\hline
\end{tabular}

SM: salários mínimos; NA**: não se aplica; FA: Frequência absoluta; FR: Frequência relativa. Fonte: Dados da pesquisa e Lopes (2018). 
A Tabela 3 apresenta o perfil antropométrico, pressórico, lipídico, glicêmico e circunferência abdominal de adolescentes nascidos a termo e pré-termo. Quando se compara o perfil antropométrico, as maiores frequências são de indivíduos eutróficos, com semelhanças nas proporções entre os grupos, porém a frequência de adolescentes com excesso de peso chama atenção em ambos. Na categoria magreza, a maior frequência foi observada entre adolescentes nascidos PT $(\mathrm{p}<0,031)$.

Ao serem analisados os percentis do perfil pressórico, isoladamente (PASM e PADM), observa-se que ambos os grupos encontravam-se classificados como adequado à idade, apresentando discreta elevação na frequência de PASM, no grupo AT (p>0,05). Quanto à classificação do percentil geral da PA, os dois grupos apresentaram frequências desejáveis em maior proporção, no entanto, 35\% dos adolescentes do grupo AT e 30\% do grupo PT apresentaram elevação na PA (p $<0,0001)$.

Observa-se que as concentrações de triglicerídeos foram mais elevadas em ambos os grupos, com frequência superior no grupo AT $(\mathrm{p}<0,0001)$.

O perfil glicêmico foi caracterizado como euglicêmico em ambos os grupos. No entanto, o grupo AT apresentou concentrações glicêmicas elevadas mais frequentes em comparação ao grupo PT (p = 0,0834).

Com relação ao percentil da CA, em sua maioria, os adolescentes foram classificados como dentro da normalidade. Contudo, observa-se que os adolescentes do grupo AT apresentaram valores elevados mais frequentes, quando comparados ao grupo PT (p<0,0001).

Tabela 3 - Perfil antropométrico, pressórico, lipídico, glicêmico e circunferência abdominal de adolescentes nascidos a termo ( $\mathrm{N}=150)$ e pré-termo ( $\mathrm{N}=50)$. Cascavel-PR, Brasil, 2019.

\begin{tabular}{|c|c|c|c|c|}
\hline Variáveis & Categorias & $\begin{array}{l}\text { A Termo- } \\
\text { FA }(\text { FR\% })\end{array}$ & $\begin{array}{l}\text { Pré-termo - } \\
\text { FA }(\text { FR\%) }\end{array}$ & p-valor \\
\hline \multirow{4}{*}{ Escore Z } & Eutrófico & $98(65 \%)$ & $31(62 \%)$ & \multirow{4}{*}{$<0,031$} \\
\hline & Magreza & $1(1 \%)$ & $4(8 \%)$ & \\
\hline & Sobrepeso & $32(21 \%)$ & $11(22 \%)$ & \\
\hline & Obesidade & $19(13 \%)$ & $4(8 \%)$ & \\
\hline \multirow{2}{*}{$\begin{array}{l}\text { Percentil da circunferência } \\
\text { abdominal }\end{array}$} & Normal & $118(79 \%)$ & $45(90 \%)$ & \multirow{2}{*}{$<0,0001$} \\
\hline & Elevado & $32(21 \%)$ & $5(10 \%)$ & \\
\hline \multirow{2}{*}{ Percentil da PASM } & Normal & $118(79 \%)$ & $45(90 \%)$ & \multirow{2}{*}{0,074} \\
\hline & Elevado & $32(21 \%)$ & $5(10 \%)$ & \\
\hline \multirow{2}{*}{ Percentil da PADM } & Normal & $109(73 \%)$ & $35(70 \%)$ & \multirow{2}{*}{0,7161} \\
\hline & Elevado & $41(275 \%)$ & $15(30 \%)$ & \\
\hline \multirow{2}{*}{ Percentil da Pressão Arterial } & Normal & $97(65 \%)$ & $35(70 \%)$ & \multirow{2}{*}{$<0,0001$} \\
\hline & Elevado & $53(35 \%)$ & $15(30 \%)$ & \\
\hline \multirow{2}{*}{ Glicemia } & Normal & $131(87 \%)$ & $48(96 \%)$ & \multirow{2}{*}{0,0834} \\
\hline & Elevado & $19(13 \%)$ & $2(4 \%)$ & \\
\hline \multirow{3}{*}{ Colesterol Total } & Desejável & $115(77 \%)$ & $38(76 \%)$ & \multirow{3}{*}{0,2197} \\
\hline & Elevado & $35(23 \%)$ & $11(22 \%)$ & \\
\hline & Não realizado & $0(0 \%)$ & $1(2 \%)$ & \\
\hline \multirow{3}{*}{ Triglicerídeos } & Desejável & $37(25 \%)$ & $29(58 \%)$ & \multirow{3}{*}{$<0,0001$} \\
\hline & Elevado & $113(75 \%)$ & $20(40 \%)$ & \\
\hline & Não realizado & $0(\%)$ & $1(2 \%)$ & \\
\hline
\end{tabular}

PASM: pressão arterial sistólica média; PADM: pressão arterial diastólica média; FA: Frequência absoluta; FR: Frequência relativa. Fonte: Dados da pesquisa e Lopes (2018).

\section{Discussão}

A caracterização da amostra evidenciou que, apesar de a maioria dos adolescentes do estudo realizarem atividades de lazer ativo, boa parte ainda representa aqueles com hábitos de vida sedentários e que realizam atividades físicas em quantidade considerada inadequada, destacando-se, entre eles, o grupo PT. Pinho e colaboradores (2017) evidenciaram alta prevalência de 
excesso de peso e elevada proporção de atividades de tempo de tela entre os adolescentes, em que $39,1 \%$ desses realizavam três ou mais atividades de tela por dia, resultado semelhante ao nosso, em que $36 \%$ do grupo AT e $25 \%$ do PT desenvolvia atividades passivas. Em revisão sistemática, Pelegrini e colaboradores (2021) apontaram uma variação na prevalência de obesidade em crianças e adolescentes brasileiros entre 3,8\% a 24\%, observando que fatores socioeconômicos, hereditários e comportamentais, incluindo atividades físicas, tempo de tela, hábitos alimentares, entre outros, estiveram associados à este resultado.

Em nosso estudo, apesar de os hábitos de tela, refeições e tipo de alimentação terem sido classificados como intermediários, os hábitos de tela inadequados se destacaram entre os AT, assim como, os hábitos alimentares mais saudáveis dentre os PT. Os hábitos alimentares classificados como restritos foram os mais frequentes entre os adolescentes no Rio Grande do Sul, indicando baixo consumo de todas os grupos alimentares e maior consumo de feijão e refrigerante. Assim como, a frequência de consumo de alimentos ultraprocessados e hipercalóricos, como doces, refrigerantes e batata frita, foi elevada (Correa, et al., 2017). Em estudo realizado no estado do Sergipe, 44,4\% dos adolescentes estavam expostos a comportamentos sedentários por mais de 3 horas ao dia, com uma maior prevalência no sexo feminino (60,2\%) (Cunha, et al., 2022). Nos municípios acima de 100.000 habitantes, mais de 70\% dos adolescentes passam duas horas ou mais em frente às telas, com a maior prevalência na região Sul. Além disso, cerca de 56,6\% realizam as refeições quase sempre ou sempre em frente à televisão, e 39,6\% consomem petiscos em frente às telas com a mesma regularidade, principalmente entre os adolescentes de escolas públicas, sendo tal comportamento possivelmente relacionado ao grau de instrução e à rotina de trabalho dos pais (Oliveira, et al., 2016). A prevalência do sedentarismo no Brasil entre os adolescentes foi de 67,8\%, assim como excesso de peso de $24,2 \%$, podendo-se apontar uma necessidade de reestruturação das políticas públicas, voltadas à promoção de estilo de vida mais saudáveis à esse grupo (Ferreira \& Andrade, 2021).

A análise do tempo de aleitamento materno, em nosso estudo, constatou que os adolescentes foram amamentados até acima de seis meses de idade. Com relação ao excesso de peso, esteve presente em cerca de 34\% no grupo AT e 30\% entre os PT. Valdés e colaboradores (2018) apontaram que a obesidade central em adolescentes apresentou correlação inversamente proporcional à duração da amamentação, indicando o aleitamento materno exclusivo como fator de proteção contra o excesso de peso e obesidade na adolescência.

Além disso, indivíduos que passaram por alguma restrição no crescimento uterino ou na infância apresentam perfil metabólico que propicia acumulação de gordura, quando associado a excessos alimentares e hábitos sedentários de vida, podendo levar a doenças cardiovasculares (DCV), como a HAS, futuramente (Hoffman, 2014). Maiores valores de IMC estiveram associados à maior PA, em ambos os sexos, tanto na infância como adolescência (Gialamas, et al., 2018).

Estudo nacional, realizado com adolescentes de 12 a 17 anos, apontou cerca de $24 \%$ com PA elevada, assim como $25 \%$ com excesso de peso, com maiores prevalências na região Sul, exceto a maior frequência de obesidade encontrada entre os adolescentes de 15 a 17 anos da região Sudeste. O estudo associa ainda maiores frequências de hipertensão à obesidade (Bloch, et al., 2016; Tozo, et al., 2020). Em Vitória - ES, diferenças significativas na PAD estiveram associadas à obesidade. Dentre os indivíduos classificados como eutróficos, $18,5 \%$ do sexo masculino e $21,2 \%$ do sexo feminino estavam hipertensos ou considerados como limítrofe, assim como, entre os indivíduos obesos, $26,3 \%$ era do sexo masculino e $25 \%$ do sexo feminino, ressaltando o aumento da frequência entre os adolescentes com excesso de peso (Cordeiro, et al., 2016). Frequências superiores foram encontradas em nosso estudo, com alteração significativa no grupo AT (35\%) e entre os PT (30\%).

Em nosso estudo observam-se alterações importantes, principalmente com relação às concentrações de triglicerídeos, que teve maior frequência dentre os AT. Níveis elevados de dislipidemias foram identificados entre adolescentes (CT=20,1\%, $\mathrm{TG}=7,8 \%$, lipoproteína de baixa densidade - $\mathrm{LDLc}=3,5 \%$ e lipoproteína de alta densidade - HDLc baixo=46,8\%). Na região Sul do Brasil, a prevalência de CT elevado foi de $22,8 \%$, resultado semelhante ao nosso, porém, a prevalência de TG elevados 
foi inferior (8,2\%) (Faria-Neto, 2016), o que pode ser em parte decorrente à divergência dos valores de referência seguidos, já que diferentes classificações e definições podem ser encontradas. Estudo realizado com crianças e adolescentes no estado do Rio Grande do Sul apontou que 34,7\% dos participantes apresentaram hipercolesterolemia (Calliari, et al., 2019). Assim como, no estado de Goiás, 36,7\% das crianças e adolescentes na faixa etária entre 10 e 12 anos, apresentaram dislipidemias, sendo que em 13,3\% esteve associado à obesidade (Guedes, et al., 2022).

Os efeitos tardios da prematuridade em mulheres, na adolescência e fase adulta, não mostraram diferenças de peso, altura e IMC entre os grupos PT e de referência, porém a relação cintura-quadril foi maior no grupo prematuro, indicando importante fator de risco para obesidade abdominal, assim como DCV na adolescência e vida adulta (Kaczmarczyk, et al., 2018). A relação da obesidade abdominal e alterações lipídicas também foi observada em estudo realizado com adolescentes na região sudeste do Brasil (Enes \& Silva, 2018).

Gialamas e colaboradores (2018) não encontraram associação entre o peso, ao nascer, ou IG de nascimento com fatores de risco cardiovasculares, em adolescentes de 11 e 18 anos de idade, contrapondo-se ao estudo de revisão sistemática que evidenciou que o IMC, o perímetro da cintura e a relação cintura-estatura são considerados fatores de risco cardiometabólico na infância e adolescência (Quadros, et al., 2017).

Nosso estudo encontrou então maiores índices de CA, PA e TG alterados no grupo AT, bem como, o grupo PT apresentou maior prevalência de atividades físicas inadequadas, maior escolaridade dos pais, maior vínculo empregatício e receberam mais benefícios sociais.

O desenho transversal pode ser uma limitação do estudo, pois avalia uma exposição em um único momento, bem como o tipo da amostra ter sido de conveniência. Contudo, os resultados apontam que adolescentes nascidos AT e PT precisam de um olhar mais atento dos profissionais de saúde, no seguimento de sua saúde, para que ações e intervenções relacionadas à redução do sedentarismo, tempo em telas, melhores hábitos alimentares e realização de atividades físicas, considerados hábitos de vida saudáveis, possam ser estimulados e implementadas, com fim de reduzir o excesso de peso e o risco de desenvolver futuras DCV. Desse modo, indicando a necessidade de estudos futuros que analisem em longo prazo essa faixa etária, bem como estudos de intervenção clínica para poder analisar efeitos sobre a saúde do adolescente.

\section{Considerações Finais}

Não foram verificadas diferenças entre os perfis antropométrico, pressórico e glicêmico entre os grupos. Alterações no perfil lipídico encontradas foram maiores entre os AT, podendo-se inferir que, apesar da prematuridade ser considerada fator de risco para doenças crônicas, os hábitos de vida podem se constituir em fator agravante para os indivíduos AT, sobrepondose à prematuridade.

\section{Referências}

Addinsoft. (2017). Software XLSTAT Versão Anual 2017.19.02. Licença ID 43894 (Node-loc).

Bloch, K. V., Klein, C. H., Szklo, M., KuschnirIII, M. C. C., Abreu, G. A., \& Barufaldi, L. A. (2016). ERICA: prevalências de hipertensão arterial e obesidade em adolescentes brasileiros. Rev Saúde Pública, 50(1):9s. https://doi.org/10.1590/S01518-8787.2016050006

Brasil. (2012). Ministério da Saúde. Saúde da criança: crescimento e desenvolvimento. Cadernos de Atenção Básica. Brasília: Ministério da Saúde.

Brasil. (2021). Ministério da Saúde. Secretaria de Vigilância em Saúde. Departamento de análise em saúde e vigilância de doenças não transmissíveis. Saúde Brasil 2020/2021: Uma análise da situação de saúde e da qualidade da informação. Brasília: Ministério da Saúde. http://bvsms.saude.gov.br/bvs/publicacoes/saude_brasil_2020_2021_situacao_saude.pdf

Burggren, W. W., \& Mueller, C. A. (2015). Developmental Critical Windows and Sensitive Periods as Three-Dimensional Constructs in Time and Space. Physiol Biochem Zool, 88(2):91-102. https://www.journals.uchicago.edu/doi/10.1086/679906

Calliari, S. S., Grando, L. G., Bertol, C. D., \& Siqueira, L. O. (2019). Dislipidemias em crianças e adolescentes do município de Marau-RS. Cad. Saúde Colet., 27(4):368-373. http://dx.doi.org/10.1590/1414-462X201900040004 
Carneiro, C. S., Peixoto, M. R. G., Mendonça, K. L., Póvoa, I. T. I. R., Nascente, I. F. M. N., Jardim, T. S. V. et al. (2017). Excesso de peso e fatores associados em adolescentes de uma capital brasileira. Rev Bras Epidemiol., 20(2):260-273. https://www.scielo.br/scielo.php?script=sci_arttext\&pid=S1415790X2017000200260\&lng=pt\&tlng=pt

Chawanpaiboon, S., Vogel, J. P., Moller, A. B., Lumbiganon, P., Petzold, M., Hogan, D., et al. (2019). Global, regional, and national estimates of levels of preterm birth in 2014: a systematic review and modelling analysis. Lancet Glob Health, 7:37-46.

Cheng, H. G. \& Phillips, M. R. (2014). Secondary analysis of existing data: opportunities and implementation. Shanghai Archives os Psychiatry, 26(6):371375. https://www.ncbi.nlm.nih.gov/pmc/articles/PMC4311114/

Cordeiro, J. P., Dalmaso, S. B., Anceschi, A. S., Sá, F. G. S., Ferreira, L. G., Cunha, M. R. H., et al. (2016). Hipertensão em estudantes da rede pública de Vitória/ES: influência do sobrepeso e obesidade. Rev Bras Med Esporte, 22(1):59-65. http://dx.doi.org/10.1590/1517-86922016220113430

Corrêa, R. S. C., Vencato, P. H., Rockett, F. C., \& Bosa, V. L. (2017). Padrões alimentares de escolares: existem diferenças entre crianças e adolescentes? Ciênc Saúde Colet., 2(2):553-562. https://doi.org/10.1590/1413-81232017222.09422016

Cunha, I. F. F., Silva, R. J. S., Ribeiro, D. S. S., Lima, E. O., Santos, J. B. N., Santos, L. S., \& Menezes, A. S. (2022). Associação entre exposição ao comportamento sedentário, indicadores de sono e fatores comportamentais em adolescentes. Research, Society and Development, 11(1):e46311125213. http://dx.doi.org/10.33448/rsd-v11i1.25213

Enes, C. C., \& Silva, J. R. (2018). Associação entre excesso de peso e alterações lipídicas em adolescentes. Ciênc Saúde Colet., 23(12):40554063. http://dx.doi.org/10.1590/1413-812320182312.27882016

Faria-Neto, J. R., Bento, V. F. R., Baena, C. P., Olandoski, M., Gonçalves, L. G. O., \& Abreu, G. A. (2016). ERICA: prevalência de dislipidemia em adolescentes brasileiros. Rev Saúde Pública, 50(1):10s. http://dx.doi.org/10.1590/S01518-8787.20160500067

Ferreira, C. S., \& Andrade, F. B. (2021). Desigualdades socioeconômicas associadas ao excesso de peso e sedentarismo em adolescentes brasileiros. Ciência e Saúde Coletiva, 26(3):1095-1104. http://dx.doi.org/10.1590/1413-81232021263.09022019

Guedes, M. R., Moura, A. M., Barros-Silveira, M., Oliveira, A. C. C. P., Caldeira, D. M., \& Bernardes, V. (2022). Dislipidemias em crianças e adolescentes de diferentes faixas etárias residentes no município de Goiânia. Research, Society and Development, 11(1):e25711124671. http://dx.doi.org/10.33448/rsdv11i1.24671

Gialamas, A., Kinnell, A., Mittinty, M. N., Davison, B., Singh, G., \& Lynch, J. (2018). Association of anthropometric measures and cardiovascular risk factors in children and adolescents: Findings from the Aboriginal Birth Cohort study. PLOS ONE, 13(6):e0199280. https://doi.org/10.1371/journal.pone.0199280

Hoffman, D. J. (2014). Growth retardation and metabolic programming: implications and consequences for adult health and disease risk. $J$ Pediatr., 90(4):325328. https://doi.org/10.1016/j.jped.2014.04.005

IDF. International Diabetes Federation. (2006). The IDF consensus worldwide de nation of the metabolic syndrome. Brussels: International Diabetes Federation.

Kaczmarczyk, K., Pituch-Zdanowska, A., Wiszomirska, I., Magiera, A., \& Ronikier, A. (2018). Long-term effects of premature birth on somatic development in women through adolescence and adulthood. $J$ Int Med Res., 46(1):44-53. http://dx.doi.org/10.1177/0300060517714369

Lopes, M. N. (2018). Relação da prematuridade com o perfil antropométrico e metabólico de adolescentes em seu contexto de vida. Cascavel: Universidade Estadual do Oeste do Paraná. Dissertação de Mestrado em Biociências e Saúde.

Luu, T. M., Katz, S. L., Leeson, P., Thébaud, B., \& Nuyt, A. M. (2016). Preterm birth: risk factor for early-onset chronic diseases. CMAJ, 188(10):736-746. https://doi.org/10.1503/cmaj.15045

Oliveira, J. S., Barufaldi, L. A., Abreu, G. A., LeaI, V. S., Brunken, G. S., Sandra Mary Vasconcelos, S. M. L., et al. (2016). ERICA: uso de telas e consumo de refeições e petiscos por adolescentes brasileiros. Rev Saúde Pública, 50(1):7s. https://doi.org/10.1590/S01518-8787.2016050006680

Pelegrini, A., Bim, M. A., Souza, F. U., Kilim, K. S. S., \& Pinto, A. A. (2021). Prevalence of overweight and obesity in Brazilian children and adolescentes: a systematic review. Rev Bras Cineantropom Desempenho Hum., 23:e80352. https://doi.org/10.1590/1980-0037.2021v23e80352

Pereira, A. S.., Shitsuka, D. M., Parreira, F. J., \& Shitsuka, R. (2018). Metodologia da Pesquisa Científica. UFSM, Lic_Computacao_Metodologia-PesquisaCientifica.pdf (ufsm.br)

Pinho, M. G. M., Adami, F., Benedet, J., \& Vasconcelos, F. A. G. (2017). Association between screen time and dietary patterns and overweight/obesity among adolescents. Rev Nutri., 30(3):377-389. https://doi.org/10.1590/1678-98652017000300010

Quadros, T. M. B., Gordia, A. P., \& Silva, L. R. (2017). Antropometria e fatores de risco cardiometabólico agrupados em jovens: revisão sistemática. Rev Paul Pediatr., 35(3):340-350. http://dx.doi.org/10.1590/1984-0462/;2017;35;3;00013

R Development Core Team. (2019). R: A Language and Environment for Statistical Computing. R Foundation for Statistical Computing Vienna, Austria: R Foundation for Statistical Computing.

Roche. (2014a). Roche Diagnostics GmbH. Accutrend Cholesterol cobas®. Mannheim, Germany.

Roche. (2014b). Roche Diagnostics GmbH. Accutrend Triglycerides cobas®. Mannheim, Germany.

SBAC. Sociedade Brasileira de Análises Clínicas. (2016). Consenso Brasileiro para a Normatização da Determinação Laboratorial do Perfil Lipídico. Brasília: Sociedade Brasileira de Análises Clínicas. 
Research, Society and Development, v. 11, n. 3, e16411326322, 2022

(CC BY 4.0) | ISSN 2525-3409 | DOI: http://dx.doi.org/10.33448/rsd-v11i3.26322

SBC. Sociedade Brasileira de Cardiologia. (2005). I Diretriz Brasileira de Diagnóstico e Tratamento da Síndrome Metabólica. Arq Bras Cardio., 84(1):1-28.

SBD. Sociedade Brasileira de Diabetes. (2019). Diretrizes da sociedade brasileira de diabetes. São Paulo: Editora Clannad.

SINASC. Sistema de Informação Sobre Nascido Vivo. (2019). Dados nascidos vivos residentes em Cascavel. http://tabnet.datasus.gov.br/cgi/tabcgi.exe?sinasc/cnv/nvuf.def

Tozo, T. A., Pereira, B. O., Menezes Junior, F. J., Montenegro, C. M., Moreira, C. M., Moreira, C. M. M., \& Leite, N. (2020). Medidas Hipertensivas em escolares: Risco da obesidade central e efeito protetor da atividade física moderada-vigorosa. Arq Bras Cardiol., 115(1):42-49. https://doi.org/10.36660/abc.20180391

UNICEF. Fundo das Nações Unidas Para a Infância. (2019). Dados do UNICEF: Monitorando a situação de crianças e mulheres. https://data.unicef.org/topic/child-survival/under-five-mortality/

Valdés, J. M. B., Alonso, M. C. V., Viltres, M. A., Pieiga, E. M., \& Pelegrin, T. S. T. (2018). Excesso de peso y obesidad central y su relación con la duración de la lactancia materna exclusiva. Rev Cubana Pediatr., 90(4):e345. http://scielo.sld.cu/scielo.php?script=sci_arttext\&pid=S003475312018000400007

Vezzoni, C. (2015). Secondary analysis in the social sciences and its relation to futures studies. On the horizon, 23(2). https://www.emeraldinsight.com/doi/abs/10.1108/OTH-02-2015-0006

WHO. World Health Organization. (2017). Global Accelerated Action for the Health of Adolescents (AA-HA!). Guidance to Support Country Implementation: World Health Organization. 